\title{
Crystallization of Amorphous Silicon Films Using Joule Heating
}

\author{
Jae-Sang Ro* \\ Department of Materials Science and Engineering, Hongik University, Seoul 121-791, Korea
}

(Received February 6, 2014 ; revised February 19, 2014 ; accepted February 22, 2014)

\begin{abstract}
Joule heat is generated by applying an electric filed to a conductive layer located beneath or above the amorphous silicon film, and is used to raise the temperature of the silicon film to crystallization temperature. An electric field was applied to an indium tin oxide (ITO) conductive layer to induce Joule heating in order to carry out the crystallization of amorphous silicon. Polycrystalline silicon was produced within the range of a millisecond. To investigate the kinetics of Joule-heating induced crystallization (JIC) solid phase crystallization was conducted using amorphous silicon films deposited by plasma enhanced chemical vapor deposition and using tube furnace in nitrogen ambient. Microscopic and macroscopic uniformity of crystallinity of JIC poly-Si was measured to have better uniformity compared to that of poly-Si produced by other methods such as metal induced crystallization and Excimer laser crystallization.
\end{abstract}

Keywords : Crystallization, Poly-Si, AMOLED, Joule heating

\section{Introduction}

Active matrix organic light emitting diode (AMOLED) has recently come into the spotlight for its applicability to the next-generation flat panel displays. Since the device operates in a current-driven mode uniform source/drain current is critical for uniform picture quality. Low temperature polycrystalline silicon is thus preferred to a-Si for the thin-film-transistor backplanes. A crystallization technology should produce poly-Si having a uniform grain size over the whole panel especially for AMOLED application. The methods of forming polycrystalline silicon at a low temperature include solid phase crystallization (SPC) $)^{1)}$, metal induced crystallization (MIC) ${ }^{2}$, metal induced lateral crystallization (MILC) $)^{3)}$, and Excimer laser crystallization $(\text { ELC })^{4}$.

Several attempts have been made to anneal silicon films by applying an electric field during the crystallization of amorphous silicon films. By applying an electric field during MILC, Jun et al. attempted to fabricate polycrystalline silicon thin film transistors using field-aided lateral crystallization ${ }^{5}$. Electric field

\footnotetext{
${ }^{*}$ Corresponding author. E-mail : jsang@hongik.ac.kr
}

enhanced silicide mediated crystallization has also been reported for low temperature crystallization ${ }^{6,7}$. In such methods, an electric field is applied to an aSi film which has an ultrathin Ni layer during the crystallization process. Sameshima et al. proposed a crystallization method with pulsed electrical currentinduced heating of silicon films. In coincidence with the voltage pulse, samples were irradiated with a $28-n s$ pulsed $\mathrm{XeCl}$ excimer laser. The authors reported that the melt duration time of the silicon thin films could be controlled using electrical current intensity in order to obtain large crystalline grain growth ${ }^{8,9)}$. They also reported the rapid crystallization of silicon films using Joule heating of metal films ${ }^{10}$. Rapid Joule heating at an intensity of about $1.0 \mathrm{MW} / \mathrm{cm}^{2}$ was demonstrated by allowing current to flow in chromium metal strips located above a-Si films. This particular method involved the achievement of crystallization by melting silicon films.

We previously reported a crystallization method named as Joule heating induced crystallization (JIC) ${ }^{11}$. In this technique, Joule heat is generated by applying an electric filed to a conductive layer located beneath or above the amorphous silicon film, and is used to raise the temperature of the silicon film to crystallization 
temperature. For the crystallization of a-Si films to occur, the Joule heat generated must be used mainly in raising the temperature of the film to its crystallization temperature. Thus, an electric field should be applied to the conductive layer for a very short time in order to generate intense pulses of energy which are conducted to the film, therefore minimizing the heating of glass substrate. As the Joule heat is generated uniformly throughout the conductive layer, the temperature of the film can be regarded as being more uniform than that achieved using other conventional heating methods. Crystallization is accomplished within the range of a millisecond. In this work we investigated crystallization kinetics of JIC and crystalline uniformity of JIC polySi.

\section{Experimental Procedure}

Schematic diagram for the JIC process is shown in Fig. 1, where an electric pulse is imposed on the specimen with ammeter and voltmeter for in-situ measurements. Fig. 1 shows an instance in which a conductive layer is located beneath the amorphous silicon films. A conductive layer such as $\mathrm{Mo}, \mathrm{Cr}$, or indium tin oxide (ITO) and a $\mathrm{SiO}_{2}$ dielectric layer are successively formed on the dielectric layer produced on the glass substrate, which is then followed by the formation of an amorphous silicon thin film thereon.

Using the plasma enhanced chemical vapor deposition (PECVD) method, a $\mathrm{SiO}_{2}$ layer (first dielectric layer) with a thickness of $300 \mathrm{~nm}$ was formed on a $0.7 \mathrm{~mm}$ thick glass substrate. An ITO thin film (conductive layer) having a thickness of $70 \mathrm{~nm}$ was deposited on the first dielectric layer by sputtering, and then a $\mathrm{SiO}_{2}$ layer (second dielectric layer) having a thickness

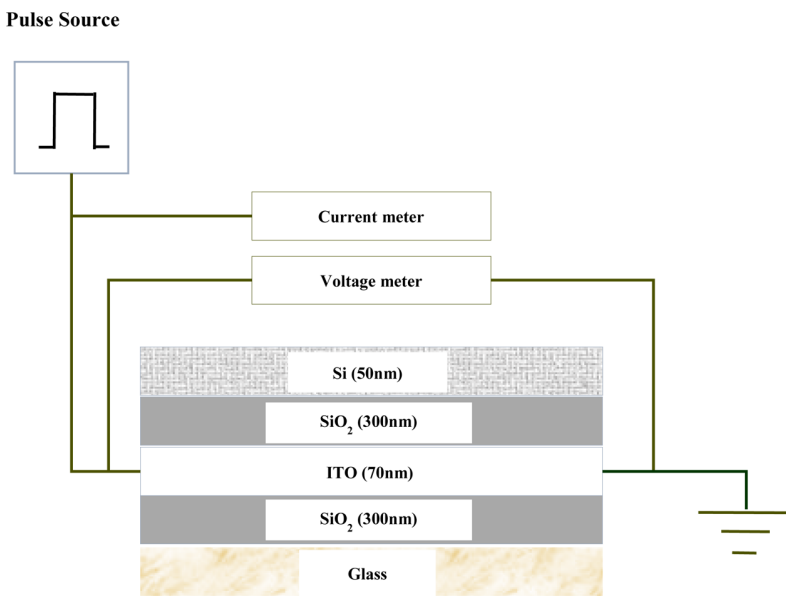

Fig. 1. Schematic diagram of experimental setup for Joule-heating induced crystallization.

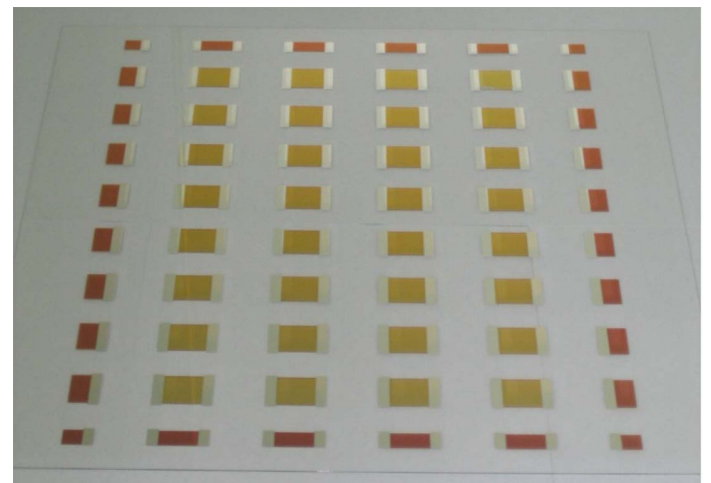

Fig. 2. Small-sized $(20 \mathrm{~mm} \times 20 \mathrm{~mm}) \mathrm{JIC}$ crystallized samples on the $2^{\text {nd }}$ generation glass substrate.

of $500 \mathrm{~nm}$ was deposited thereon using the PECVD method. The PECVD method also resulted in an amorphous silicon thin film having a thickness of $50 \mathrm{~nm}$ being deposited on the second dielectric layer. Thus, an array, including the amorphous silicon thin film shown in Fig. 1, was prepared. Fig. 2 shows small-sized crystallized samples using JIC method on the $2^{\text {nd }}$ generation glass substrate (Corning Eagle $2000^{12)}$ ). Overall dimensional size of each specimen was $20 \times 30 \mathrm{~mm}^{2}$, where the crystallization window and each electrode at both sides were $20 \times 20 \mathrm{~mm}^{2}$ and $5 \times 20 \mathrm{~mm}^{2}$, respectively.

The sheet resistance of the ITO conductive layer was measured to be $30 \Omega / \square$. An electric field was applied to an ITO film within the range of a millisecond for crystallization. The microstructure of crystallized films was determined by transmission electron microscopy (TEM). Macroscopic uniformity of crystallinity of JIC poly-Si samples was measured using Raman spectroscopy and was compared to that of poly-Si produced by the methods of MIC and ELC, respectively. A Jasco-NR110 Raman system was used at room temperature with the $514.5 \mathrm{~nm}$ line from an $\mathrm{Ar}$ ion laser. Raman shift was measured between $350 \mathrm{~cm}^{-1}$ and $650 \mathrm{~cm}^{-1}$. Broad peak of a-Si was observed at $480 \mathrm{~cm}^{-1}$ while crystalline Si exhibited a sharp peak at $520 \mathrm{~cm}^{-1}$.

\section{Results and Discussion}

Two main factors whether crystallization occurs or not using a JIC process are power density $\left(\mathrm{W} / \mathrm{cm}^{2}\right)$ and pulsing time, respectively. Power density affects the heating rate during the period of electrical pulsing. Raman spectroscopy was employed to check if crystallization occurred according to processing conditions of power density and pulsing time. When an electric 


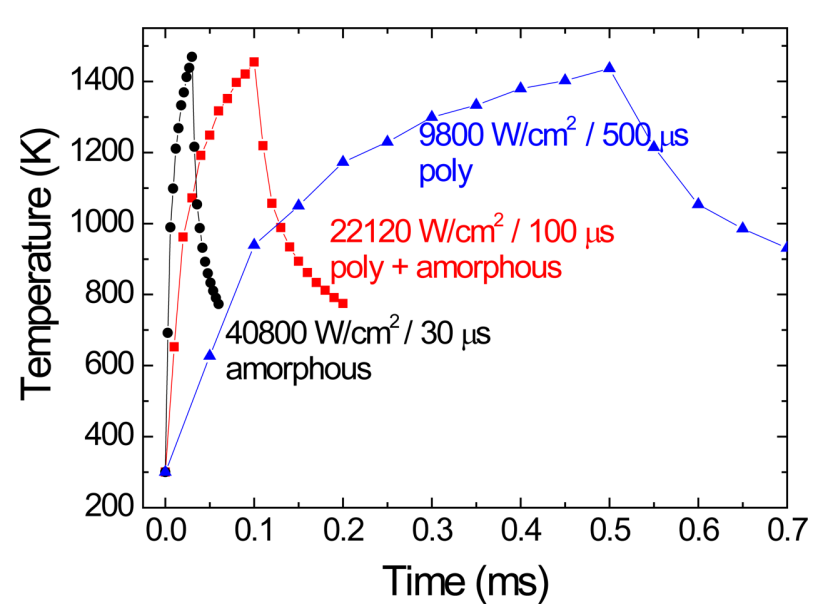

Fig. 3. The simulated temperature profile according to the processing conditions of the power density and the pulsing time.

field was applied with the power density of $40800 \mathrm{~W} /$ $\mathrm{cm}^{2}$ for $30 \mu \mathrm{s}$, crystallization was not observed to occur. The $100 \mu$ s pulse with the power density of 22120 $\mathrm{W} / \mathrm{cm}^{2}$ resulted in partial crystallization. Meanwhile, complete crystallization was observed with the power density of $9800 \mathrm{~W} / \mathrm{cm}^{2}$ for $500 \mu \mathrm{s}$. Fig. 3 indicates the simulated temperature profile under these three different processing conditions. Each thin film in the specimen had no patterned features and Joule heat was assumed to be generated uniformly in the ITO thin film. Furthermore, the major process time was about tens or hundreds of microseconds. Thus the convective and radiation heat transfer was assumed to be negligible. Based on this approximation, the temperature of the film could be estimated by the one-dimensional heat conduction model, given by

$$
\begin{aligned}
& \frac{\partial \rho C T}{\partial t}=\frac{\partial}{\partial x}\left(K \frac{\partial T}{\partial x}\right)+S \\
& S=I V / A h_{\text {ITO }}
\end{aligned}
$$

Where $\rho, C, K$ are density, specific heat, and thermal conductivity, respectively, for each layer and $S$ denotes volumetric Joule heat generation rate only in the ITO film. Here, a time-dependent input pulse of current, $I$, and voltage, $V$, was imposed on the specimen of process window area, $A$, and ITO film of thickness, $h_{\text {ITO}}$. The finite difference form of the heat conduction equation was solved by the Crank-Nicolson method. As the power density increases the heating rate increases as demonstrated in Fig. 3. Joule heating under each three condition causes almost the same peak temperature of the film to rise above $1400 \mathrm{~K}$. However, the duration above a critical temperature during the period of heating (during electrical pulsing) and cooling (after electrical pulsing) is different according to processing parameters, which affects the crystallization kinetics.

In order to investigate the temperature dependence of the crystallization kinetics, we conducted SPC experiments using silicon wafers that had the structure of a 50 nm-thick PECVD a-Si / 500 nm-thick $\mathrm{SiO}_{2} /$ silicon wafer. Solid phase crystallization was conducted using a tube furnace in nitrogen ambient at temperatures ranging from $600^{\circ} \mathrm{C}$ to $1000^{\circ} \mathrm{C}$. Crystallization kinetics was checked as a function of annealing temperature and time using Raman spectroscopy. While the crystallization process was completed in $20 \mathrm{hrs}$ at $600^{\circ} \mathrm{C}$,

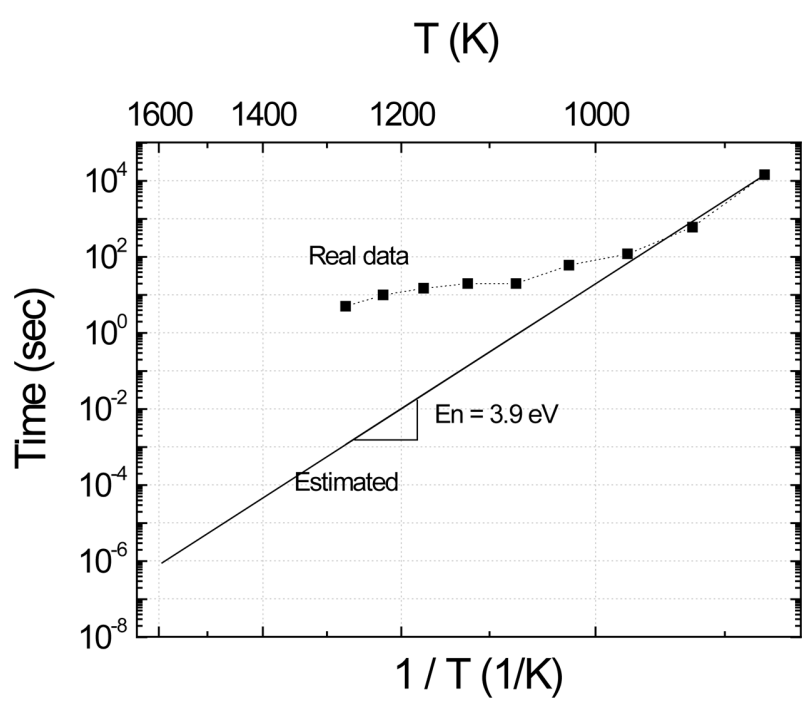

Fig. 4. Arrhenius plot of the measured incubation time. Notice that the measured data does not fit to straight line. The estimated straight line was obtained using the measured data at low temperatures and using the value of nucleation activation energy of $3.9 \mathrm{eV}$.

Table 1. Measured and estimated incubation time vs. crystallization temperatures

\begin{tabular}{|c|c|c|}
\hline & $\begin{array}{c}\text { Measured } \\
\text { incubation time }\end{array}$ & $\begin{array}{c}\text { Estimated } \\
\text { incubation time }\end{array}$ \\
\hline $600^{\circ} \mathrm{C}$ & $\sim 4 \mathrm{hrs}$ & $4 \mathrm{hrs}$ \\
\hline $700^{\circ} \mathrm{C}$ & $<2 \mathrm{~min}$ & $1.16 \mathrm{~min}$ \\
\hline $800^{\circ} \mathrm{C}$ & $<20 \mathrm{sec}$ & $912 \mathrm{~ms}$ \\
\hline $900^{\circ} \mathrm{C}$ & $<15 \mathrm{sec}$ & $25.01 \mathrm{~ms}$ \\
\hline $1000^{\circ} \mathrm{C}$ & $<5 \mathrm{sec}$ & $1.2 \mathrm{~ms}$ \\
\hline $1050^{\circ} \mathrm{C}$ & N/A & $314.5 \mu \mathrm{s}$ \\
\hline $1100^{\circ} \mathrm{C}$ & N/A & $90.5 \mu \mathrm{s}$ \\
\hline $1150^{\circ} \mathrm{C}$ & N/A & $28.4 \mu \mathrm{s}$ \\
\hline $1200^{\circ} \mathrm{C}$ & N/A & $9.64 \mu \mathrm{s}$ \\
\hline $1250^{\circ} \mathrm{C}$ & N/A & $3.52 \mu \mathrm{s}$ \\
\hline $1300^{\circ} \mathrm{C}$ & N/A & $1.37 \mu \mathrm{s}$ \\
\hline & &
\end{tabular}




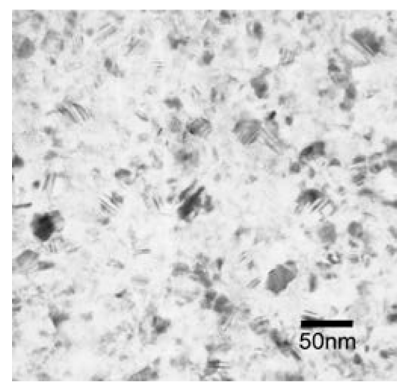

(a)

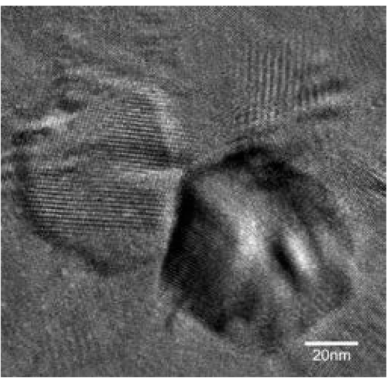

(b)

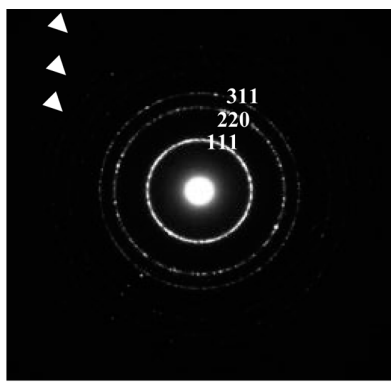

(c)

Fig. 5. (a) Bright filed TEM bright-field micrograph, (b) high resolution TEM micrograph, and (c) TED pattern of the $\mathrm{JIC}$ poly-Si crystallized at room temperature in an electric field of $800 \mathrm{~V} / \mathrm{cm}$ for $320 \mu \mathrm{s}$.

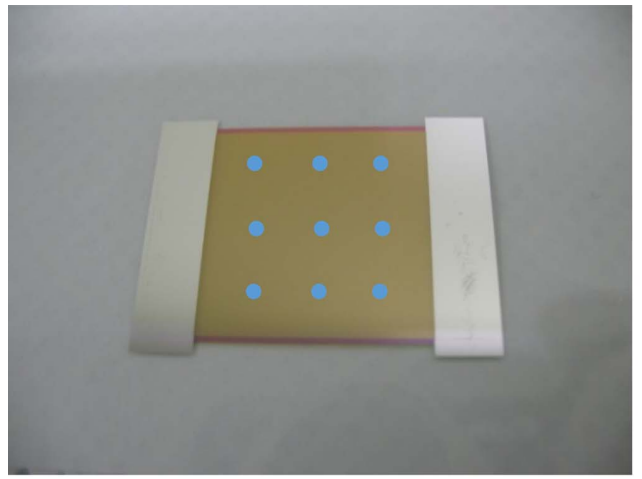

(a)

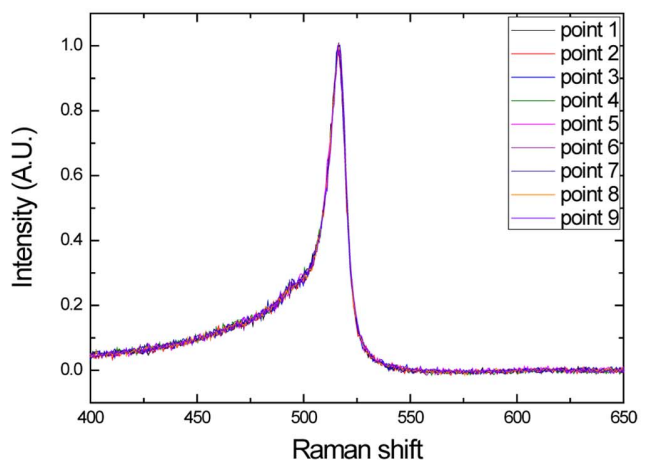

(b)

Fig. 6. (a) JIC poly-Si sample $(20 \mathrm{~mm} \times 20 \mathrm{~mm})$ showing 9 spots where Raman spectroscopy was conducted, (b) Raman spectra of JIC poly-Si analyzed from 9 different locations.

it was over in $25 \mathrm{~s}$ at $1000^{\circ} \mathrm{C}$. Since SPC kinetics is controlled by the nucleation rate, and the activation energy needed to nucleate silicon crystals has a high value of $3.9 \mathrm{eV}^{13)}$, the crystallization rate is significantly increased when the crystallization temperature is raised. Moreover, in the case of furnace annealing, the sample was found to be unable to reach the setting temperature of $1000^{\circ} \mathrm{C}$ within 25 s. Fig. 4 indicates an Arrhenius plot of an incubation time. The dotted line represents the measured data while the solid straight line denotes the estimated data using the nucleation activation energy of $3.9 \mathrm{eV}$ with the measured data under $600^{\circ} \mathrm{C}$. The measured and estimated incubation time are summarized in Table 1 at crystallization temperatures ranging from $600^{\circ} \mathrm{C}$ to $1300^{\circ} \mathrm{C}$. It can be seen that the estimated incubation time indeed becomes less than $1 \mathrm{~ms}$ at temperatures above $1000^{\circ} \mathrm{C}$. As indicated in Fig. 4 the incubation time at $1400 \mathrm{~K}$ is estimated to be around $36 \mu \mathrm{s}$, which may explain the experimental results as shown in Fig. 3.

Fig. 5(a) and Fig. 5(b) show a TEM bright field micrograph and high resolution TEM micrograph, and Fig. 5(c) shows the transmission electron diffraction (TED) pattern of the JIC poly-Si. The a-Si film is fully crystallized at room temperature in an electric field of $800 \mathrm{~V} / \mathrm{cm}$ for $320 \mu \mathrm{s}$. The radii of the TED patterns shown in Fig. 5(c) for the JIC poly-Si have been indexed and found to correspond to the interplanar spacing of silicon. The grains are preferentially oriented with the (111), (220) and (311) directions according to the TED patterns. The microstructure of the polycrystalline silicon thin film exhibits a nano-crystalline phase. The polycrystalline silicon produced has a very small grain size of $\sim 30 \mathrm{~nm}$ and exhibits grains of equiaxed morphology uniformly distributed in terms of the grain size as can be seen in Fig. 5(b). Such a microstructure cannot be obtained by solid state transformation using conventional heat-treatment methods since crystallization is completed during the period of heating-up. In order to check the macroscopic crystallineuniformity Raman spectroscopy ${ }^{14)}$ was conducted on the 9 spots in the JIC poly-Si sample having dimension of $20 \times 20 \mathrm{~mm}^{2}$ as demonstrated in Fig. 6(a). Fig. 6(b) shows Raman spectra measured at 9 different locations. 
Crystalline non-uniformity of JIC poly-Si was measured to be $\sim 1.1 \%$ while ELC poly-Si was measured to have crystalline non-uniformity of $\sim 2.3 \%$ and $\sim 7.2 \%$ for MIC poly-Si. Thus, the macroscopic uniformity of the grain size of the JIC poly-Si was found to be excellent in addition to the microscopic one.

\section{Conclusions}

This study demonstrated the possibility of achieving the microsecond crystallization of amorphous Si thin film via Joule heating. Such a process does not make use of any metallic element for preferential nucleation, and is completed within a millisecond at room temperature. Poly-Si films featuring nano-crystalline sized grains were obtained by the present crystallization method. Crystalline non-uniformity of JIC poly-Si was measured to be $\sim 1.1 \%$ while ELC poly-Si was measured to have crystalline non-uniformity of $\sim 2.3 \%$ and $\sim 7.2 \%$ for MIC poly-Si. As microscopic and the macroscopic uniformity of the grain size of the JIC poly-Si film is excellent, this process is expected to find its applications, especially with regards to AMOLED. In order to apply JIC method in mass production line where large-sized glass substrate is used thickness uniformity of a conductive layer should be improved.

\section{Acknowledgments}

This work was supported by the IT R\&D program of MOTIE/KEIT. [10043413, Development of Heat Treatment Processes for Fabrication of Next-Generation
Flat Panel Displays and Semiconductor Devices Using Rapid Heating Methods] and JIC samples were fabricated in Samsung Display.

\section{References}

1. A. T. Voutsas, M. K. Hatalis, J. Electrochem. Soc., 139 (1992) 2659.

2. R. S. Wagner, W. C. Ellis, Appl. Phys. Lett., 4 (1964) 89.

3. S.-W. Lee, S.-K. Joo, IEEE Electron Dev. Lett., 17 (1996) 160.

4. J. S. Im, H. J. Kim, M. O. Thompson, Appl. Phys. Lett., 63 (1993) 2969.

5. S.-I Jun, Y.-H. Yang, J.-B. Lee, D.-K. Choi, Appl. Phys. Lett., 75 (1999) 2235.

6. J. Jang, J. Y. Oh, S. K. Kim, Y. J. Choi, S. Y. Yoon, C. O. Kim, Nature, 395 (1998) 481.

7. S. Y. Yoon, J. Y. Oh, C. O. Kim, J. Jang, J. Appl. Phys., 84 (1998) 6463.

8. T. Sameshima, K. Ozaki, N. Andoh, Appl. Phys., A 71 (2000) 1.

9. T. Sameshima, N. Andoh, H. Takahashi, J. Appl. Phys., 89 (2001) 5362.

10. T. Sameshima, Y. Kaneko, N. Andoh, Appl. Phys., A 73 (2001) 419.

11. J.-S. Ro, W.-E. Hong, SID Digest of Technical Papers (2006) 1280.

12. Samsung Corning Precision Glass Inc., www. samsungscp.co.kr.

13. I.-W. Wu, A. Chiang, M. Fuse, L. Öveçoglu, T. Y. Huang, J. Apply. Phys., 65 (1989) 4036.

14. W.-E. Hong, J.-S. Ro, J. Appl. Phys., 114 (2013) 073511. 